\title{
Ruscogenin alleviates cognitive dysfunction by inhibiting the activation of isoflurane-induced NLRP3 inflammasome in aged mice
}

\author{
Xiaohu Liang ${ }^{1}$ Xiaoqun Luo ${ }^{1}$, Danping Li $^{1}$, Lingqiong Kong ${ }^{2 *}$ \\ ${ }^{1}$ Department of Anesthesiology, Pingxiang People's Hospital, Pingxiang City, Jiangxi Province, China; ${ }^{2}$ Department \\ of Anesthesiology, Hainan General Hospital (Hainan Affiliated Hospital of Hainan Medical University), Haikou City, \\ Hainan Province, China
}

*Corresponding author: Lingqiong Kong, Department of Anesthesiology, Hainan General Hospital (Hainan Affiliated Hospital of Hainan Medical University), No. 19 Xiuhua Road, Haikou City, Hainan Province 570311, China. Email: lqkong7153@163.com

Received: 7 September 2021; Accepted: 1 October 2021; Published: 12 November 2021 (C) 2021 Codon Publications

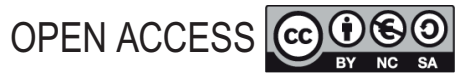

ORIGINAL RESEARCH

\begin{abstract}
Ruscogenin exerts an anti-inflammatory effect in the pathogenesis of various human diseases, including pulmonary hypertension, acute lung injury, acute pancreatitis and cerebral ischemia. Its role in isoflurane-induced rats with postoperative cognitive dysfunction (POCD) was investigated in this study. Aged rats were exposed to isoflurane for establishing a model of POCD, and administered with ruscogenin by gavage. Cognitive dysfunction was evaluated by the Morris water maze test. Hematoxylin and Eosin (H\&E) staining was designed to assess neuronal damage. Markers of brain damage and neuroinflammation were detected by enzyme-linked-immunosorbent serologic assay. Isoflurane exposure caused impaired cognitive function by increasing escape latency, decreasing the time taken for crossing target and time in target quadrant. However, administration of ruscogenin reversed these cognitive dysfunctions. Abnormal morphological phenomena on neurons and enhanced levels of serum calcium-binding protein $\beta$ (S-100 $\beta$ ) and neuron-specific enolase (NSE) were identified in mice post-isoflurane exposure. Administration of ruscogenin ameliorated the neuronal morphological damages and reduced the levels of S-100 $\beta$ and NSE in the hippocampi of isoflurane-induced aged mice. Ruscogenin also attenuated isofluraneinduced enhancements in the levels of Interleukin (IL)-1 $\beta$, IL-6 and tumor necrosis factor-alpha in the hippocampi of mice. Isoflurane-induced enhancements in the mRNA expression levels of NLR family pyrin domain containing 3 (NLRP3), ASC, IL-1 $\beta$ and IL-18 proteins were also restored by administration of ruscogenin. Ruscogenin exerted neuroprotective effects against isoflurane-induced cognitive dysfunction and neuroinflammation through blocking of NLRP3 pathway.
\end{abstract}

Keywords: ruscogenin; cognitive dysfunction; isoflurane; NLRP3 inflammasome; postoperative cognitive dysfunction

\section{Introduction}

Anesthetics, such as isoflurane (ISO) and sevoflurane, are widely used in surgical operations (Alwardt et al., 2005). However, the increasing incidences of complications associated with exposure to anesthetics have been reported recently (Belcher et al., 2017). Postoperative cognitive dysfunction (POCD) is a reversible neurodegenerative disease caused by anesthesia and surgery in elderly patients. POCD includes impairments in psychomotor speed, visual and spatial ability, executive function, information processing, memory, concentration and attention (Huang et al., 2020). Owing to POCD, medical expenses are increased, length of hospitalization is prolonged, and the overall life of patient is affected by it with increasing morbidity and mortality (Cui et al., 2018). Pathogenic factors, 
including oxidative stress, neuroinflammation, decline in neurogenesis and neuron apoptosis, have been demonstrated to contribute to the pathogenesis of POCD (Shao et al., 2020). Therefore, drugs or rehabilitation therapies that regulate balance in neurotransmitters, cause reduction in neuroinflammation, and result in the suppression of neuron apoptosis, have been widely used in the treatment of POCD (Wang et al., 2021a).

Ruscogenin (RUS), the primary effective steroid sapogenin discovered in Ophiopogon japonicus, was first isolated from Ruscus aculeatus, which has been widely used in the treatment of acute and chronic inflammatory diseases (Lu et al., 2014a). For example, ruscogenin exerted antifibrotic and anti-inflammatory effects to attenuate streptozotocin-induced diabetic nephropathy (Lu et al., 2014b). Monocrotaline-induced pulmonary hypertension (Bi et al., 2013), cerulein-induced acute pancreatitis (Ercan et al., 2019) and particulate matter-induced acute lung injury (Wang et al., 2021b) were ameliorated by ruscogenin through its anti-inflammatory capacity. Cerebral ischemic-induced inflammatory pathway was also suppressed by ruscogenin (Guan et al., 2013), and ruscogenin alleviated ischemia/reperfusion-induced blood-brain barrier dysfunction (Zhang et al., 2020). Since surgery is indicated to promote the secretion of pro-inflammatory factors and blood-brain barrier dysfunction to induce neuroinflammation during the development of POCD (Hovens et al., 2016), the role of ruscogenin in anesthetic-induced POCD was investigated in this study.

Isoflurane, a halogenated hydrocarbon inhalation anesthetic, induces neuropathological sensitive tissue lesions, inflammation and apoptosis of hippocampal cells, and is also depicted to exert neurotoxic effects on the nervous system (Lin \& Zuo, 2011). Isofluraneinduced cognitive dysfunction and neuroinflammation were used to construct the animal model of POCD (Cao et al., 2018). This study investigated the effects of ruscogenin on isoflurane-induced cognitive dysfunction and neuroinflammation as well as demonstrated the underlying mechanism.

\section{Materials and methods}

\section{Animal model}

A total of 80 male Sprague-Dawley mice (80-week old; weight 250-280 g) were purchased from SLAC Laboratory Animal Technology Co. Ltd. (Shanghai, China). The animals were maintained individually in pathogen-free cages with free access to water and food. The study was approved by the Ethics Committee of Pingxiang People's Hospital, and was in accordance with
National Institutes of Health Laboratory Animal Care and Use Guidelines. The animals were grouped as follows: control $(\mathrm{N}=20)$, isoflurane (ISO, $\mathrm{N}=20)$, ISO + $1 \mathrm{mg} / \mathrm{kg}$ ruscogenin (RUS, $\mathrm{N}=20$ ), and $\mathrm{ISO}+3 \mathrm{mg} / \mathrm{kg}$ ruscogenin $(\mathrm{N}=20)$. Mice in the control group received room air; those in the ISO group were exposed to $2 \%$ isoflurane (Sigma-Aldrich, San Francisco, CA, USA) for $4 \mathrm{~h}$; and the ones in the ISO + RUS groups were first exposed to isoflurane and then administered 1 or $3 \mathrm{mg} / \mathrm{kg}$ of ruscogenin (J\&K Scientific Ltd., Beijing, China) through intragastric route. Five rats in each group were decapitated under anesthesia of $35 \mathrm{mg} / \mathrm{kg}$ sodium pentobarbital. After $24 \mathrm{~h}$ of ruscogenin administration, the animal brains were harvested and conducted with the following analyses. The remaining 15 rats in each group were subjected to the Morris water maze test (MWM).

\section{Morris water maze test}

After $24 \mathrm{~h}$ post-administration of ruscogenin, mice were trained in a circular tank (tank diameter: $150 \mathrm{~cm}$ and height: $70 \mathrm{~cm}$ ) with hidden platform (diameter: $10 \mathrm{~cm}$ ), which was located $2 \mathrm{~cm}$ below the surface of water. Mice were placed in four equal hypothetical quadrants with four points (North, East, South and West), and were subjected to swim to record the time taken (latency) to find the hidden platform by video camera mounted above the water maze. Mice were trained for five days, and the platform was removed on the sixth day to test route length to find platform, time taken for crossing target, and time in target quadrant for $2 \mathrm{~min}$. The data were recorded and analyzed by the image analysis system connected to the camera.

\section{Hematoxylin and Eosin (H\&E) staining}

The hippocampi of brain tissues isolated from mice were immersed in 4\% paraformaldehyde, and then embedded in paraffin. The tissues were sliced into $4-\mu \mathrm{m}$ sections, and the sections were used for Hematoxylin and Eosin staining before prior to microscopic observations (Leica Microsystems, Wetzlar, Germany).

\section{Enzyme-Linked-Immunosorbent Serologic Assay (ELISA)}

The hippocampi were incubated with RIPA lysis buffer (Beyotime, Beijing, China) and centrifuged at 12,000 $\mathrm{g}$ for $1 \mathrm{~h}$. The supernatant was harvested, and the protein concentration was determined by Pierce ${ }^{\text {Tw }}$ BCA Protein Assay Kit (Thermo Fisher Scientific, Waltham, MA, USA). Levels of serum calcium-binding protein $\beta$ (S-100 $\beta)$, neuron-specific enolase (NSE), Interleukin (IL)-1 $\beta$, IL-6 and tumor necrosis 
factor-alpha (TNF)- $\alpha$ were measured using commercial kits (Nanjing Biotechnology Co. Ltd., Nanjing, China).

\section{Western blot test}

Protein samples from hippocampi were separated by $10 \%$ sodium dodecyl sulfate-polyacrylamide gel electrophoresis (SDS-PAGE), and transferred onto nitrocellulose membrane. The membrane was blocked and probed with specific antibodies: anti-NLR family pyrin domain containing 3 (NLRP3), anti-apoptosis-associated speck-like protein (ASC) (1:2,000; Abcam, Cambridge, UK), antiIL-1 $\beta$, anti-IL-18 (1:2,500; Abcam) and anti- glyceraldehyde 3-phosphate dehydrogenase (GAPDH) (1:3,000; Abcam). Following incubation with horseradish peroxidase-conjugated secondary antibody (1:4,000; Abcam) and tetramethylbenzidine, the protein bands were visualized using chemiluminescence (Sigma-Aldrich).

\section{Statistical analysis}

All data in at least triple replicates were expressed as mean \pm SEM, and analyzed by Student's $t$-test or one-way analysis of variance (one-way ANOVA) under the SPSS software. $P<0.05$ was considered as statistically significant.

\section{Results}

\section{Ruscogenin ameliorated isoflurane-induced cognitive dysfunction}

Mice were exposed to isoflurane, and the Morris water maze test demonstrated that the representative route length of mice to find the platform increased in the isoflurane group as compared to that in the control group (Figure 1A). However, administration of ruscogenin decreased the route length in the isoflurane group (Figure 1A). Moreover, the escape latency was enhanced in rats post-isoflurane exposure (Figure 1B), while ruscogenin reduced the escape latency (Figure 1B). Isoflurane-induced impairment of memory, which was evidenced by the decreased time taken for crossing target (Figure 1C) and time in target quadrant (Figure 1D), was raised by the administration of ruscogenin. Therefore, ruscogenin improved the long-term spatial learning and ability to memorize in isoflurane-treated mice.

\section{Ruscogenin ameliorated isoflurane-induced neuronal damage}

Morphological analysis of the hippocampi demonstrated a relatively large cell body in the control group

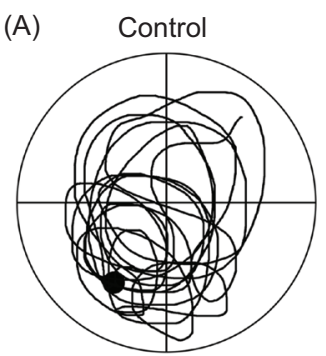

(B)

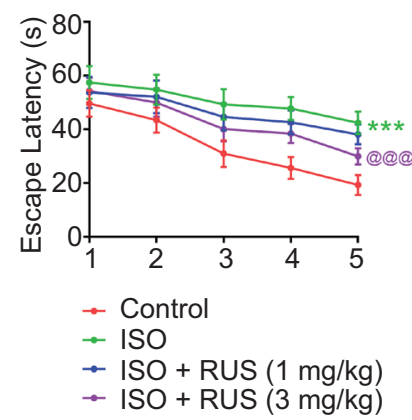

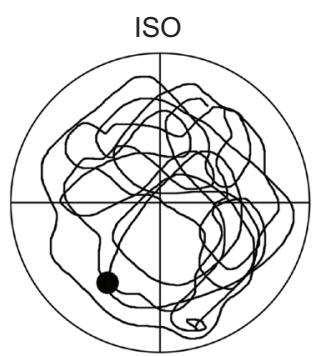

(C)
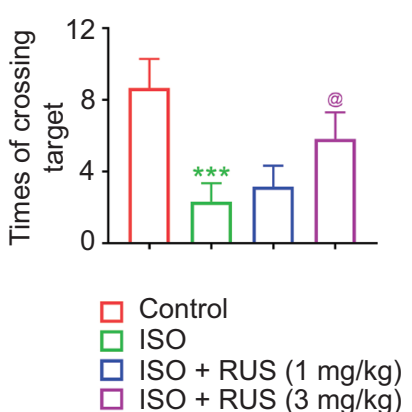
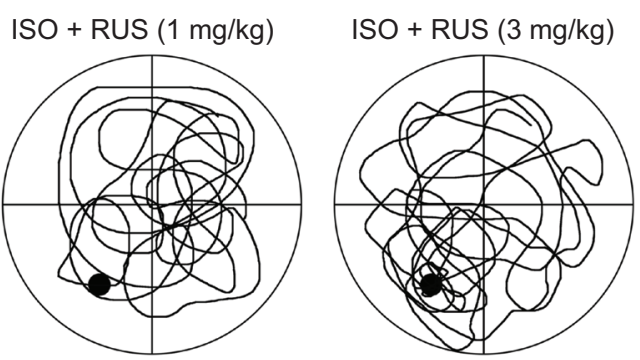

(D)

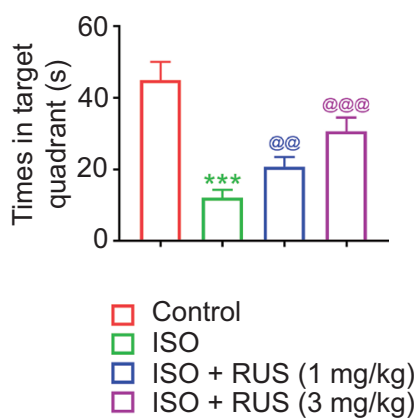

Figure 1. Ruscogenin ameliorated isoflurane-induced cognitive dysfunction. (A) Ruscogenin administration decreased the route length to find the platform in rats post-isoflurane exposure. (B) Ruscogenin administration attenuated isoflurane-induced increase of escape latency in rats. Data were expressed as mean \pm SEM. (C) Ruscogenin administration attenuated isoflurane-induced decrease in time for crossing target by rats. Data were expressed as mean \pm SEM. (D) Ruscogenin administration attenuated isoflurane-induced decrease in time by rats in target quadrant. Data were expressed as mean \pm SEM. ${ }^{* * *}$ vs. control group, $P<0.001$. @, @@, @@@vs. ISO, $P<0.05, P<0.01, P<0.001$. 
compared to the isoflurane group (Figure 2A). Exposure to isoflurane induced shrunken cell bodies, empty vesicles, dark cytoplasm and pyknotic nuclei in the neurons (Figure 2A). Administration of ruscogenin reduced these abnormal phenomena in the neurons (Figure 2A). Additionally, isoflurane enhanced the levels of brain damage biomarkers, S-100 $\beta$ and NSE, which were reversed by ruscogenin (Figure $2 \mathrm{~B}$ ). These results established that ruscogenin protected hippocampal neurons against isoflurane-induced neuronal damage in aged mice.

\section{Ruscogenin ameliorated isoflurane-induced inflammation}

Levels of pro-inflammatory factors-IL-1 $\beta$, IL- 6 and TNF- $\alpha$ - were enhanced in the neurons of the hippocampus of rats post-isoflurane exposure (Figure 3). However, administration of ruscogenin reduced the levels of IL-1 $\beta$, IL-6 and TNF- $\alpha$ (Figure 3) to protect hippocampal neurons against isoflurane-induced neuroinflammation in aged mice.

\section{Ruscogenin suppressed NLRP3 pathway}

Isoflurane exposure increased the protein expressions of NLRP3, ASC, IL-1 $\beta$ and IL-18 in the hippocampus (Figure 4), while administration of ruscogenin reduced their protein expressions (Figure 3) to protect hippocampal neurons against isoflurane-induced NLRP3 activation in aged mice.

\section{Discussion}

Chinese medicinal herbs, with antioxidant, antiinflammatory and antiapoptotic capacities, have been widely used for the prevention of cognitive dysfunction of POCD (Chu et al., 2018). Ophiopogon japonicus has been demonstrated as a pharmacological Chinese medicinal herb in distinct diseases, including diabetes, cancers and inflammatory diseases (Chen et al., 2016). Since ruscogenin, the primary effective steroid sapogenin from Ophiopogon japonicus, has been reported to ameliorate ischemic-induced inflammatory pathway (Guan et al., 2013) or blood-brain barrier dysfunction (Zhang et al., 2020), its role in POCD was investigated in the present study.

A former study has pointed that cognitive dysfunction caused by anesthetics is a challenging problem for post-surgery patients (Belrose and Noppens, 2019). Patients with POCD suffer from abstract thinking, decreased social activities and persistent impairment of memory (Johnson et al., 2002). It has been reported that isoflurane increased the incidence of POCD in patients compared to propofol (Geng et al., 2017), and also
(A)
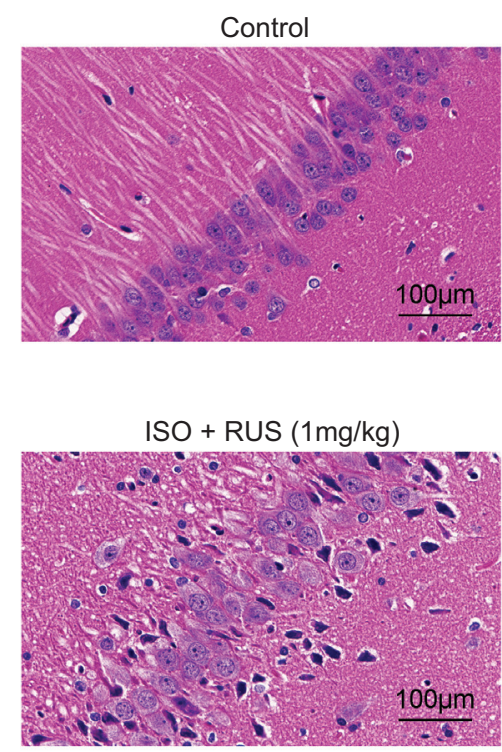

ISO

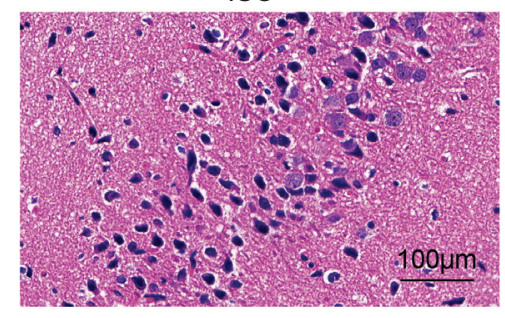

ISO + RUS (3mg/kg)

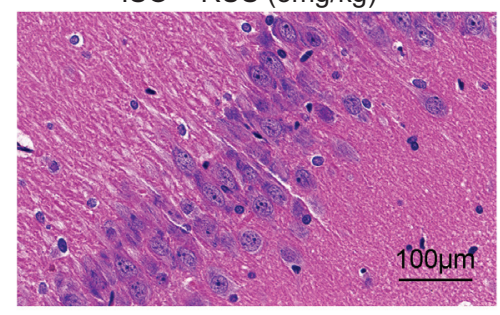

(B)
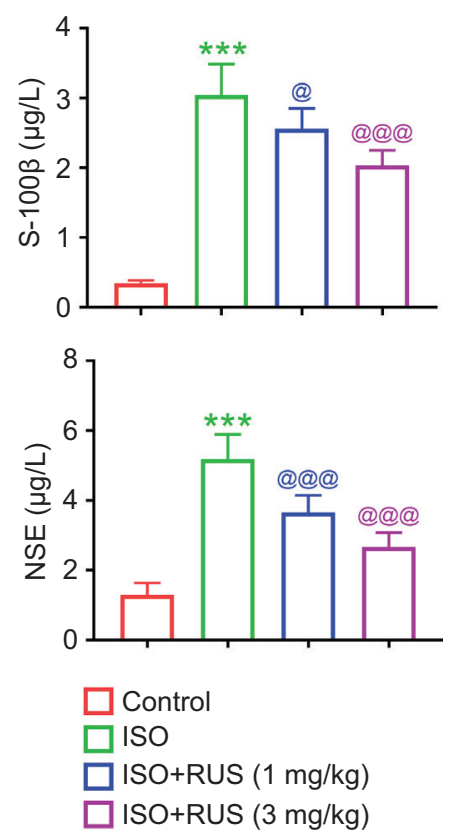

Figure 2. Ruscogenin ameliorated isoflurane-induced neuronal damage. (A) Isoflurane exposure induced shrunken cell bodies, empty vesicles, dark cytoplasm and pyknotic nuclei in the neurons compared to the control, while ruscogenin administration reduced these abnormal phenomena in the neurons. Scale bars $=100 \mu \mathrm{m}$. (B) Ruscogenin administration attenuated isoflurane-induced enhancements in the levels of S-100 $\beta$ and NSE in rats. ${ }^{* *}$ vs. control, $P<0.001$. @, @@@vs. ISO, $P<0.05, P<0.001$. 

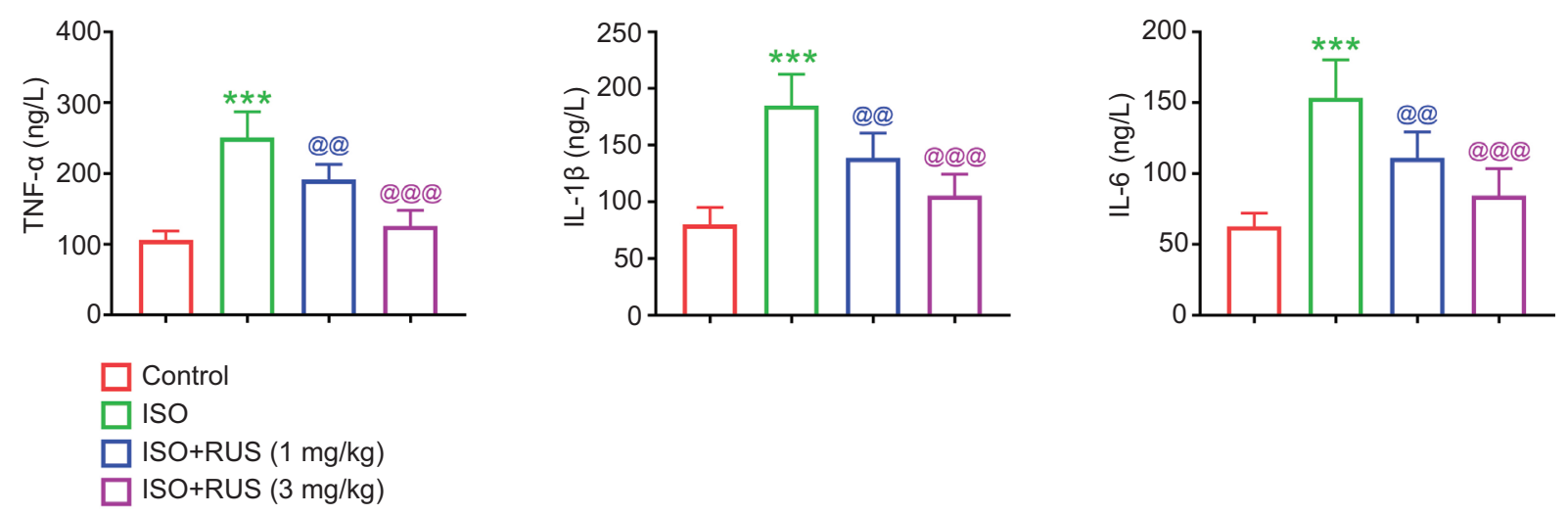

Figure 3. Ruscogenin ameliorated isoflurane-induced inflammation. Ruscogenin administration attenuated isoflurane-induced enhancements in IL-1 $\beta$, IL-6 and TNF- $\alpha$ levels in rats. " vs. control, $P<0.001$. @@,@@ $@$ vs. ISO, $P<0.01, P<0.001$.
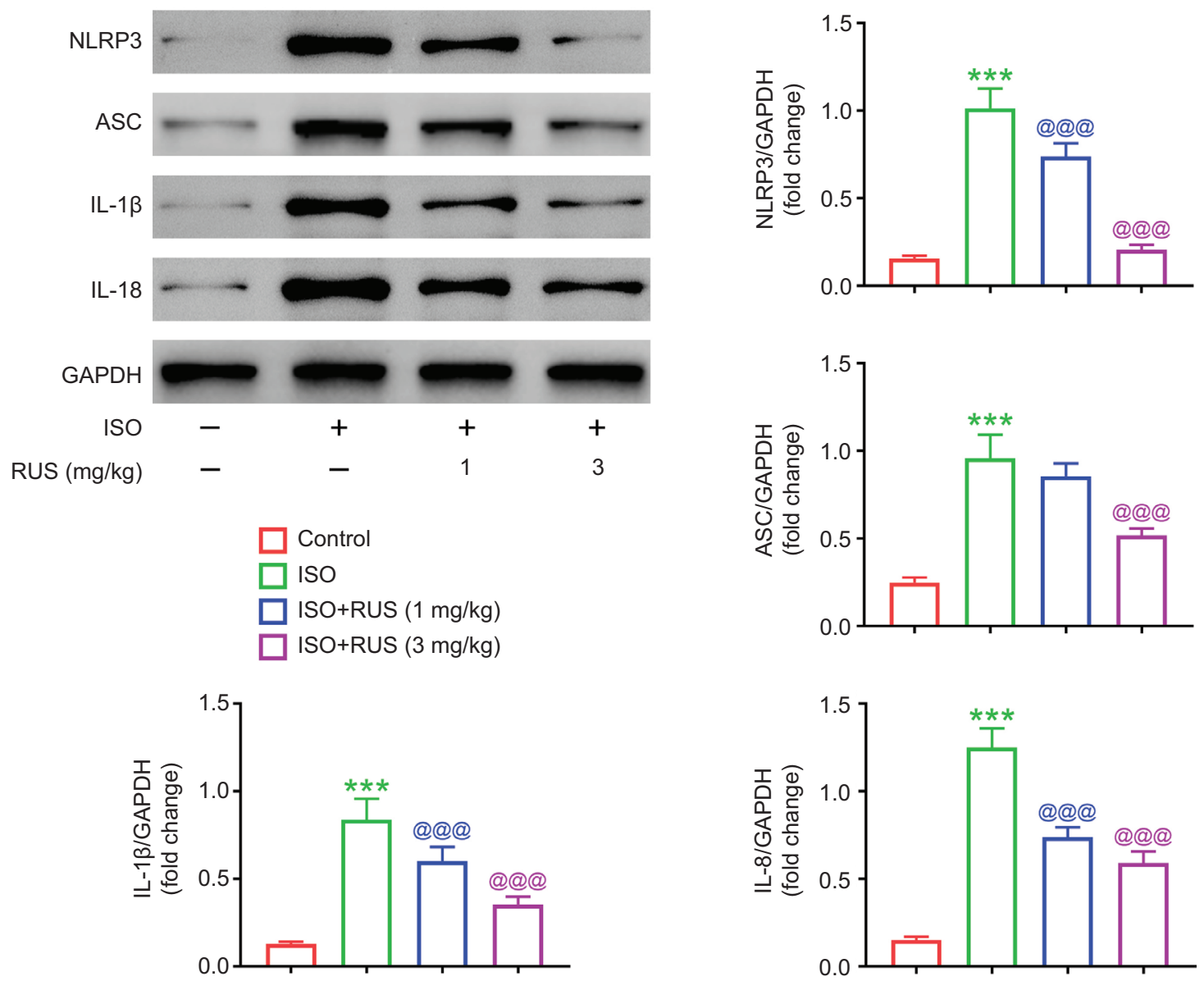

Figure 4. Ruscogenin suppressed NLRP3 pathway. Ruscogenin administration attenuated isoflurane-induced enhancements in the mRNA expression levels of NLRP3, ASC, IL-1 $\beta$ and IL-18 in rats. " vs. control, $P<0.001$. @@@vs. ISO, $P<0.001$.

induced cognitive deficits in aged mice (Wu et al., 2015). In this study, mice were exposed to isoflurane to establish a model of POCD. Results indicated that isoflurane induced cognitive impairment in aged mice, while ruscogenin reversed the results by reducing escape latency, time taken for crossing target and time in target quadrant, thereby indicating its role in improving long-term spatial learning and memory abilities in isoflurane-treated mice. Moreover, abnormal morphological phenomena in the neurons were also observed in the hippocampi of mice post-isoflurane exposure. The plasma levels of both S-100 $\beta$ and $A \beta 1-40$ proteins were upregulated in POCD 
patients post-isoflurane (Geng et al., 2017). This study has consistently demonstrated that isoflurane upregulated S-100 $\beta$ and NSE levels in aged mice. However, administration of ruscogenin attenuated neuronal damage and reduced levels of S-100 $\beta$ and NSE, suggesting that ruscogenin protected hippocampal neurons against isoflurane-induced neuronal damage in aged mice.

Increasing evidence has indicated that surgery or anesthesia-induced neuroinflammation is the major contributor of POCD (Safavynia and Goldstein, 2019). Levels of IL-1 $\beta$, IL-6, and TNF- $\alpha$ were significantly upregulated in POCD patients exposed to isoflurane compared to those exposed to propofol (Geng et al., 2017). Isoflurane induced neuroinflammation, thus contributing to cognitive impairment in aged mice (Cao et al., 2018). Ruscogenin reduced the levels of TNF- $\alpha$ and IL-1 $\beta$ to attenuate cerebral ischemic injury (Guan et al., 2013). Here, ruscogenin also attenuated isoflurane-induced increase in the levels of IL-1 $\beta$, IL- 6 and TNF- $\alpha$ in aged mice, thus exerting anti-inflammatory effect against POCD. Oxidative stress is implicated in the pathogenesis of POCD, and strategies to modulate oxidative stress and inflammation have been widely used in the mitigation of POCD (Ho et al., 2020). The potential antioxidant role of ruscogenin in isoflurane-induced mice must be investigated in the future studies.

NLRP3 inflammasome, containing NLRP3, ASC and caspase-1, functions as an innate immune sensor and participates in cell death, including pyroptosis, ferroptosis, necroptosis and apoptosis (Huang et al., 2021). Aberrant activation of NLRP3 inflammasome is involved in inflammatory diseases, Alzheimer's disease, diabetes and cancers (Huang et al., 2021). Overactivation of NLRP3 inflammasome contributes to the progression of POCD (Zhao et al., 2021). NLRP3 inflammasome regulates the activation of caspase- 1 and mediates the maturation and secretion of IL-1 $\beta$ and IL-18, thus associating with neuroinflammation and oxidative stress during progression of POCD (Wei et al., 2019). Activation of NLRP3 inflammasome was also found to be associated with isoflurane-induced cognitive impairment and inflammation in aged mice (Wang et al., 2018). Suppression of NLRP3 inflammasome activation attenuated isoflurane-induced pyroptosis and cognitive impairment (Fan et al., 2018). Importantly, ruscogenin suppressed the activation of NLRP3 inflammasome to ameliorate blood-brain barrier dysfunction caused by cerebral ischemia (Cao et al., 2016). This study established that increased protein expression levels of NLRP3, ASC, IL-1 $\beta$ and IL-18 in the hippocampi of mice post-isoflurane exposure were reversed by administration of ruscogenin. Therefore, administration of NLRP3 inflammasome inhibitor, ruscogenin, might have ameliorated isoflurane-induced cognitive impairment and reduced pyroptosis in aged mice.
In summary, ruscogenin demonstrated neuroprotective effect against isoflurane-induced cognitive impairment and neuroinflammation via repression of NLRP3/ caspase-1 pathway, thus providing a novel therapeutic strategy for POCD treatment.

\section{Competing Interests}

The authors state that there were no conflicts of interest to disclose.

\section{Author Contribution}

Xiaohu Liang designed the study and supervised data collection. Xiaoqun Luo analyzed and interpreted the data. Danping Li and Lingqiong Kong prepared and reviewed the draft of manuscript for publication. All authors read and approved the final manuscript.

\section{References}

Alwardt, C.M., Redford, D. and Larson, D.F., 2005. General anesthesia in cardiac surgery: a review of drugs and practices. Journal of Extra-Corporeal Technology 37: 227-235. PMID: 16117465

Belcher, A., Leung, S., Cohen, B., Yang, D., Mascha, E., Turan, A., et al. 2017. Incidence of complications in the post-anesthesia care unit and associated healthcare utilization in patients undergoing non-cardiac surgery requiring neuromuscular blockade 2005-2013: a single center study. Journal of Clinical Anesthesia 43: 33-38. https://doi.org/10.1016/j.jclinane.2017.09.005

Belrose, J.C. and Noppens, R.R., 2019. Anesthesiology and cognitive impairment: a narrative review of current clinical literature. BMC Anesthesiology 19: 241. https://doi.org/10.1186/ s12871-019-0903-7

Bi, L., Zhu, R., Kong, H., Wu, S.-l., Li, N., Zuo, X., et al. 2013. Ruscogenin attenuates monocrotaline-induced pulmonary hypertension in rats. International Immunopharmacology 16(1): 7-16. https://doi.org/10.1016/j.intimp.2013.03.010

Cao, G., Jiang, N., Hu, Y., Zhang, Y., Wang, G., Yin, M., et al. 2016. Ruscogenin attenuates cerebral ischemia-induced blood-brain barrier dysfunction by suppressing TXNIP/NLRP3 inflammasome activation and the MAPK pathway. International Journal of Molecular Sciences 17: 1418. https://doi.org/10.3390/ ijms17091418

Cao, Y., Li, Z., Ma, L., Ni, C., Li, L., Yang, N., et al. 2018. Isoflurane-induced postoperative cognitive dysfunction is mediated by hypoxia-inducible factor- $1 \alpha$-dependent neuroinflammation in aged rats. Molecular Medicine Reports 17: 7730-7736. https://doi.org/10.3892/mmr.2018.8850

Chen, M.-H., Chen, X.-J., Wang, M., Lin, L.-G. and Wang, Y.-T., 2016. Ophiopogon japonicus-a phytochemical, ethnomedicinal and pharmacological review. Journal of Ethnopharmacology 2(181): 193-213. https://doi.org/10.1016/j.jep.2016.01.037 
Chu, J.M.T., Xiong, W., Linghu, K.G., Liu, Y., Zhang, Y., Zhao, G.D., et al. 2018. Siegesbeckia Orientalis L. extract attenuates postoperative cognitive dysfunction, systemic inflammation, and neuroinflammation. Experimental Neurobiology 27: 564-573. https://doi.org/10.5607/en.2018.27.6.564.

Cui, R.-S., Wang, K. and Wang, Z.-L., 2018. Sevoflurane anesthesia alters cognitive function by activating inflammation and cell death in rats. Experimental and Therapeutic Medicine 15(5): 4127-4130. https://doi.org/10.3892/etm.2018.5976

Ercan, G., Tartar, R.l., Solmaz, A., Gulcicek, O.B., Karagulle, O., Meric, S., et al. 2019. Examination of protective and therapeutic effects of ruscogenin on cerulein-induced experimental acute pancreatitis in rats. Annals of Surgical Treatment and Research 97: 271-281. https://doi.org/10.4174/astr.2019.97.6.271

Fan, Y., Du, L., Fu, Q., Zhou, Z., Zhang, J., Li, G. and Wu, J., 2018. Inhibiting the NLRP3 inflammasome with MCC950 ameliorates isoflurane-induced pyroptosis and cognitive impairment in aged mice. Frontiers in Cellular Neuroscience 19(12): 426. https://doi. org/10.3389/fncel.2018.00426

Geng, Y.-j., Wu, Q.-h. and Zhang, R.-q., 2017. Effect of propofol, sevoflurane, and isoflurane on postoperative cognitive dysfunction following laparoscopic cholecystectomy in elderly patients: a randomized controlled trial. Journal of CLINICAL ANESthesia 38: 165-171. https://doi.org/10.1016/j.jclinane.2017.02.007

Guan, T., Liu, Q., Qian, Y., Yang, H., Kong, J., Kou, J. and Yu, B., 2013. Ruscogenin reduces cerebral ischemic injury via NF-kBmediated inflammatory pathway in the mouse model of experimental stroke. European Journal of Pharmacology 714: 303-311. https://doi.org/10.1016/j.ejphar.2013.07.036

Ho, Y.-S., Zhao, F.-Y., Yeung, W.-F., Wong, G.T.-C., Zhang, H.-Q. and Chang, R.C.-C., 2020. Application of acupuncture to attenuate immune responses and oxidative stress in postoperative cognitive dysfunction: what do we know so far? Oxidative Medicine and Cellular Longevity 2020: 9641904. https://doi. org/10.1155/2020/9641904

Hovens, I., Leeuwen, B., Mariani, M., Kraneveld, A. and Schoemaker, R., 2016. Postoperative cognitive dysfunction and neuroinflammation; cardiac surgery and abdominal surgery are not the same. Brain, Behavior, and Immunity 54: 178-193. https://doi.org/10.1016/j.bbi.2016.02.003

Huang, J.-M., Lv, Z.-T., Zhang, B., Jiang, W.-X. and Nie, M.-B., 2020. Intravenous parecoxib for early postoperative cognitive dysfunction in elderly patients: evidence from a meta-analysis. Expert Review of Clinical Pharmacology 13: 451-460. https://doi.org/1 0.1080/17512433.2020.1732815

Huang, Y., Xu, W. and Zhou, R., 2021. NLRP3 inflammasome activation and cell death. Cellular \& Molecular Immunology 18(9):2114-2127. https://doi.org/10.1038/s41423-021-00740-6

Johnson, T., Monk, T., Rasmussen, L., S., Abildstrom, H., Houx, P., Korttila, K., Kuipers, H. M., et al., and ISPOCD2 Investigators, 2002. Postoperative cognitive dysfunction in middle-aged patients. Anesthesiology 96: 1351-1357. https://doi. org/10.1097/00000542-200206000-00014

Lin, D. and Zuo, Z., 2011. Isoflurane induces hippocampal cell injury and cognitive impairments in adult rats. Neuropharmacology 61: 1354-1359. https://doi.org/10.1016/j.neuropharm.2011.08.011
Lu, H.-J., Tzeng, T.-F., Liou, S.-S., Chang, C.J., Yang, C., Wu, M.-C. and Liu, I.M., 2014a. Ruscogenin ameliorates experimental nonalcoholic steatohepatitis via suppressing lipogenesis and inflammatory pathway. BioMed Research International 2014: 652680. https://doi.org/10.1155/2014/652680

Lu, H.-J., Tzeng, T.-F., Liou, S.-S., Da Lin, S., Wu, M.-C. and Liu, I.M., 2014b. Ruscogenin ameliorates diabetic nephropathy by its anti-inflammatory and antifibrotic effects in streptozotocin-induced diabetic rat. BMC Complementary and Alternative Medicine 14: 110-110. https://doi. org/10.1186/1472-6882-14-110

Safavynia, S.A. and Goldstein, P.A., 2019. The role of neuroinflammation in postoperative cognitive dysfunction: moving from hypothesis to treatment. Frontiers in Psychiatry 9: 752-752. https://doi.org/10.3389/fpsyt.2018.00752

Shao, A., Fei, J., Feng, S. and Weng, J., 2020. Chikusetsu saponin IVa alleviated sevoflurane-induced neuroinflammation and cognitive impairment by blocking NLRP3/caspase-1 pathway. Pharmacological Reports 72: 833-845. https://doi.org/10.1007/ s43440-020-00078-2

Wang, C.-M., Chen, W.-C., Zhang, Y., Lin, S. and He, H.-F., 2021a. Update on the mechanism and treatment of sevofluraneinduced postoperative cognitive dysfunction. Frontiers in Aging Neuroscience 13: 702231. https://doi.org/10.3389/ fnagi.2021.702231

Wang, Z., Meng, S., Cao, L., Chen, Y., Zuo, Z. and Peng, S., 2018. Critical role of NLRP3-caspase-1 pathway in age-dependent isoflurane-induced microglial inflammatory response and cognitive impairment. Journal of Neuroinflammation 15: 109. https://doi.org/10.1186/s12974-018-1137-1

Wang, Y.-w., Wu, Y.-h., Zhang, J.-z., Tang, J.-h., Fan, R.-p., Li, F., Yu, B.-y., Kou, J.-p. and Zhang, Y.-y., 2021b. Ruscogenin attenuates particulate matter-induced acute lung injury in mice via protecting pulmonary endothelial barrier and inhibiting TLR4 signaling pathway. Acta Pharmacologica Sinica 42: 726-734. https://doi.org/10.1038/s41401-020-00502-6

Wei, P., Yang, F., Zheng, Q., Tang, W. and Li, J., 2019. The potential role of the NLRP3 inflammasome activation as a link between mitochondria ROS generation and neuroinflammation in postoperative cognitive dysfunction. Frontiers in Cellular Neuroscience 21(13): 73. https://doi.org/10.3389/ fncel.2019.00073

Wu, J., Li, H., Sun, X., Zhang, H., Hao, S., Ji, M., Yang, J. and Li, K., 2015. A mitochondrion-targeted antioxidant ameliorates isoflurane-induced cognitive deficits in aging mice. PloS One 10: e0138256. https://doi.org/10.1371/journal.pone.0138256

Zhang, Y., Hu, Y., Li, M., Wang, J., Guo, G., Li, F., Yu, B. and Kou, J., 2020. The traditional Chinese medicine compound GRS alleviates blood-brain barrier dysfunction. Drug Design, Development and Therapy 14: 933-947. https://doi.org/10.2147/ DDDT.S229302

Zhao, S., Chen, F., Wang, D., Han, W., Zhang, Y. and Yin, Q., 2021. NLRP3 inflammasomes are involved in the progression of postoperative cognitive dysfunction: from mechanism to treatment. Neurosurgical Review 44: 1815-1831. https://doi.org/10.1007/ s10143-020-01387-z 